\title{
Wissen aus Bewegungsdaten
}

\section{Bessere Stammdaten in ERP-Systemen}

\section{Dirk Schmalzried, Siegfried Schmalzried, Matthias Fiebig}

Die Erhöhung der Stammdatengüte ist ein wichtiges Ziel. Mit ihr verbunden sind geringere Fehlerkosten, zuverlässigere Prozesse, höhere Termintreue, bessere Produktqualität und Marge. Im Artikel werden einige Ideen illustriert, wie die Analyse der Bewegungsdaten zu besseren Stammdaten in ERP-Systemen führen kann. Dabei wird ein Rahmen aufgespannt, in dem verschiedene Methoden zum Einsatz kommen.

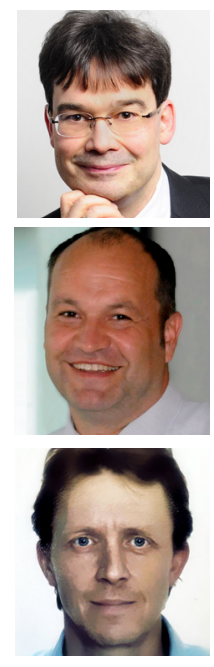

Prof. Dr. Dirk Schmalzried ist seit 2020 Professor für Wirtschaftsinformatik, insbesondere e-Business, am Fachbereich Wirtschaftsingenieurwesen der Ernst Abbe Hochschule Jena.

Prof. Dr.-Ing. Siegfried Schmalzried ist seit 2010 Professor für Automatisierungstechnik, Fertigungstechnik und Werkzeugmaschinen an der Hochschule Furtwangen.

Diplom-Informatiker Matthias Fiebig ist als Product Owner und Gruppenleiter bei der Firma ORSOFT $\mathrm{GmbH}$ verantwortlich für das Design und die Entwicklung von Software für Produktionsplanung und Stammdatenpflege.
$\mathrm{D}$ ie zunehmende Zahl anfallender und nötiger Daten, kürzere Zykluszeiten und Variantenkomplexität durch Produktkonfiguration stellen immer höhere Anforderungen an die Güte von Stammdaten. Mit den wachsenden Anforderungen entwickelt sich gleichzeitig auch das verfügbare Instrumentarium weiter, um zu besseren Stammdaten zu kommen; wie z. B. eine Datenspeicherung und schnellere Auswertung in In-MemoryDatenbanken oder KI-Methoden zur Datenanalyse. Bessere Stammdaten führen zu Wettbewerbsvorteilen, z. B. wegen höherer Kundenindividualität oder schnellerer Marktverfügbarkeit und zur Vermeidung von Verlusten

? In diesem Beitrag lesen Sie:

- wie durch eine Analyse von Bewegungsdaten die Stammdaten einer kontinuierlichen Verbesserung unterworfen werden können,

- welche Eigenschaften ein allgemeines Prozess-Rahmenwerk haben sollte,

- in welchen Anwendungsdomänen es beispielsweise zum Einsatz kommen kann. oder Risiken. So führen falsche Maße zu schlecht ausgelasteten Transporten oder ein Produkt in der falschen Verpackung zu hohen Kosten.

\section{Zielstellung}

Ausgehend von verschiedenen praktischen Beispielen soll ein verallgemeinerter Rahmen helfen, in Unternehmen einen Verbesserungsprozess zu etablieren, der in einer höheren Stammdatenqualität resultiert. Dazu werden anfallende Bewegungsdaten kontinuierlich genutzt, um einige stammdatenbezogene Kennziffern zu verbessern. Dies sind insbesondere die drei in Tabelle 1 hervorgehobenen KPIs.

\section{Fallbeispiele}

Die folgenden praktischen Beispiele illustrieren, mit welchem Vorgehen bessere Stammdaten erzielt werden können.

\section{Fallbeispiel 1: Mit mehr Daten zu einem besseren Fertigungsprozess}

Die Unternehmenspraxis zeigt einen großen Einfluss der Konstruktion auf das Produktionsergebnis. Bereits während der Konstruktion werden die Randbedingungen für die Produktion festgelegt. Dies umfasst nicht nur das Produkt selbst mit seinen qualitativen Eigenschaften, sondern auch die Prozessfähigkeit und Prozessrobustheit bei der Herstellung des Produktes bis zum Beschaffungsprozess. Mit dem Design for Manufacturing and Assembly (DFMA) wird in den frühen Phasen des Entwurfs, der Entwicklung und der Konstruktion auf eine produktionsgerechte Auslegung und Produktgestaltung geachtet. Wesentlicher Ansatzpunkt für Verbesserungen in der Produktentwicklung ist ein robustes Design für eine hohe Zuverlässigkeit (Fehlerfreiheit) der zugrundeliegenden Unternehmens- und Produktionsprozesse. Messbar ist diese Robustheit von Produkten und Prozessen indirekt, z. B. mithilfe von internen oder externen Fehlerraten. Um robuste Systeme zu schaffen, schlägt die Literatur eine Unempfindlichkeit gegen die Streuung vor. Prozesse sind robust, wenn das Prozessergebnis möglichst wenig von unvermeidlichen Schwankungen der Parameter, Materialeigenschaften 


\begin{tabular}{|l|l|}
\hline KPI & Bedeutung \\
\hline Genauigkeit & $\begin{array}{l}\text { Grad der Übereinstimmung des Stammdatums mit dem Objekt in der } \\
\text { realen Welt }\end{array}$ \\
\hline Vollständigkeit & Bis zu welchem Grad sind alle Aspekte des Realweltobjekts abgebildet? \\
\hline Aktualität & $\begin{array}{l}\text { Repräsentiert das Stammdatum die aktuellen Parameter des Objektes der } \\
\text { realen Welt? }\end{array}$ \\
\hline Konsistenz & $\begin{array}{l}\text { Grad der Übereinstimmung verschiedener Repräsentationen der Objekte } \\
\text { der realen Welt untereinander (z. B. in verschiedenen Systemen) }\end{array}$ \\
\hline Relevanz & $\begin{array}{l}\text { Zu welchem Grad eignen sich die Stammdaten für einen speziellen An- } \\
\text { wendungsfall? }\end{array}$ \\
\hline Verfügbarkeit & $\begin{array}{l}\text { Sind die Stammdaten für einen konkreten Nutzer zu einem Zeitpunkt } \\
\text { verfügbar? }\end{array}$ \\
\hline
\end{tabular}

Tabelle 1: Höhere Stammdatenqualität.

und Umgebungsbedingungen abhängt [1, 2].

Veränderliche Materialeigenschaften innerhalb von Toleranzbändern sind eine Ursache für Streuungen der Qualität. Im Zerspanungsprozess von hochlegierten Stählen beispielsweise gibt es deutliche Streuungen während der Bearbeitung. Abhängig von der Materialcharge entwickeln sich zum Beispiel bei der Zerspanung medizinischer Bauteile teilweise handhabbare und taugliche Späne, aber auch unkontrollierbare und prozessstörende Späne. Diese Information liegt in Form von Bewegungsdaten im ERP-System vor. Weiterhin wird im gleichen Zusammenhang eine deutliche Streuung des WerkzeugverschleiBes beobachtet, der auch als Bewegungsdatum im ERP-System erfasst werden kann. Erstaunlich ist dies, da die Materiallieferung aufgrund der Stammdaten jeweils identisch erfolgt und der Lieferant sich akribisch an die vorgegebenen Normen hält. Die dort zu findenden Toleranzkorridore bei Legierungsbestandteilen sind in diesem Zusammenhang ursächlich für unterschiedliches Prozessverhalten. Darüber hinaus werden einige übliche und notwendige Materialbestandteile in der Norm nicht genannt und bilden damit einen unkontrollierbaren Freiheitsgrad. Der Fertigungsbetrieb hat jedoch in der Regel kein vertiefendes werkstoffkundliches und chemisches Expertenwissen darüber, welche chemischen Bestandteile der Norm für einen stabilen Bearbeitungsprozess in einem besonders engen Toleranzkorridor gehalten werden sollten. Wird nun das Prozesswissen in Form von Bewegungsdaten aus dem PP-Modul mit dem Qualitätswissen aus dem QMModul und den Stammdaten zusammengeführt, so ermöglicht dies die Aufdeckung von Auffälligkeiten und Mustern. Sie zeigen, welche Stammdaten einen Einfluss auf das Prozessverhalten haben. Der Materialhersteller seinerseits nimmt bei jeder Materialcharge eine Materialprobe und kann daraus den tatsächlichen Anteil der Legierungsbestandteile wesentlich präziser als von der Norm gefordert für die zugehörige Charge benennen. Darüber hinaus können auch die Volumenanteile der enthaltenen nicht genormten chemischen Elemente erfasst und dem Kunden verfügbar gemacht werden. Werden nun die Stammdaten auf Kundenseite im MM-Modul mit Parametern erweitert, die dem Lieferanten bekannt sind, können mithilfe von $\mathrm{KI}$ hochinteressante Muster erkannt und der Prozess robuster geführt werden. Gleichzeitig können Erkenntnisse gewonnen werden, welche Vorgaben evtl. sogar zu eng bemessen wurden, aber im Prozess keinen störenden Einfluss haben. Diese Vorgaben können zukünftig freier und ggf. kostengünstiger gestaltet werden. Zur kontinuierlichen Prozessverbesserung lässt sich diese Vorgehensweise zusätzlich erweitern auf eine Vielzahl von Parametern und Objekten, wie Daten von Werkzeugen, Aufspannungen und Kühlschmierstoffen.

\section{Fallbeispiel 2: Mit Bewegungs- daten neue Stammdaten designen}

In den rückgemeldeten Bewegungsdaten im ERP-System sind Prozessinformationen versteckt, die für das Design der Stammdaten benutzt werden können. Ein regelhafter Verschleiß von Elementen führt z. B. zu längeren Prozesszeiten. So führt der Verschleiß von Kugeln in einer Zementmühle dazu, dass über die Lebenszeit hinweg gesehen tendenziell immer länger gemahlen werden muss, um die gleiche Feinheit zu erhalten. Wenn die anfallenden Bewegungsdaten auf solche Muster hin ausgewertet werden, können daraus bessere Arbeitspläne anhand der realen Prozesszeiten entworfen werden, welche die Realität genauer widerspiegeln. Damit wird wiederum die Produktion besser planbar, weil die Vorgabewerte in den Arbeitsplänen zeitlich genauer sind. Bei diesem Vorgehen muss ein Ausgleich aus Genauigkeit und Robustheit gefunden werden, da nicht die Prozessschwankung abgebildet werden soll, sondern das Prozessmuster oder die Prozesstendenz. Nicht jede Durchführung wird exakt die gleiche Zeit benötigen, aber ein Muster, das eine Abhängigkeit oder einen Zusammenhang mit weiteren Prozessgrößen liefert, sollte im Stammdatendesign berücksichtigt werden.

\section{Fallbeispiel 3: Aus Qualitätszahlen zu besseren Qualitätsstammdaten}

Es gibt planmäßige, reaktive und vorausschauende Wartungen. Reaktive Wartungen sind häufig mit höheren Kosten verbunden und sollen daher vermieden werden. Planmäßige Wartungen erfolgen zumeist aufgrund von Herstellervorgaben. Vorausschauende Wartungen haben den Vorteil der Vermeidung von Ausfällen, sind jedoch bezüglich ihrer Planbarkeit schlechter als planmäßige Wartungen. Indem das Wissen aus bisher erfolgten vorausschauenden Wartungen extrahiert wird und die Stammdaten für planmäßige Wartungen angepasst werden, kann eine bessere Wartungsplanung erzielt werden. In der Folge werden neben einem Wartungsplan, der eine eindeutige 
Zuordnung von Ressourcen, Personal und Zeit enthält, zusätzlich eine wahrscheinlichkeitsbasierte kapazitive Wartungsplanung, welche ausgehend von erwarteten Ausfällen in einem Intervall (nach einer Verteilungsfunktion) die zu erwartende Kapazitätslast in einem kommenden Intervall (z. B. eine Woche) ermittelt, betrachtet. So können Überlasten vermieden werden, indem entsprechende Aufträge verschoben werden. Die Voraussetzung dafür ist, dass a) in den Stammdaten als weiteres Modell eine Verteilungsfunktion hinterlegt wird und b) die Parameter dieser Funktion (also wahrscheinlichstes Intervall plus ermittelte Streuung) aus den Bewegungsdaten der vorausschauenden Wartung speist. Im Resultat wird die planmäßige Wartung verbessert.

\section{Fallbeispiel 4: Aus der Verteilung von Verkäufen zu besseren Be- standsstammdaten}

Für eine reichweitenorientierte Planung in der Produktion gibt es zwei prinzipielle Möglichkeiten: 1. den Verzicht auf mengenorientierte Stammdatenobjekte wie "Mindestbestand" und stattdessen eine permanente Auswertung der Bedarfe und darauf bezogen eine Produktions- und Beschaffungsplanung sowie -optimierung, sodass jederzeit rein planerisch eine bestimmte Bestandsreichweite in Tagen gewährleistet ist. 2. In regelmäßigen Abständen führt eine Datenanalyse der tatsächlichen Bedarfe, der Produktions- und Beschaffungssituation sowie des beeinflussenden Weltwissens (z. B. regionale Ferien, Wetter, Feiertage mit Geschenken) zu einer rollierenden Anpassung der planungsrelevanten Stammdaten. Während Fall 1 sehr gut mit ganz außergewöhnlichen Bedarfssituationen umgehen kann und Pläne häufig aktualisiert, werden im Fall 2 die Produktionspläne seltener verworfen. Er ist besser für stabilere Bedarfssituationen geeignet. Im Fall 2 werden rollierend mittels ABC-Analyse diejenigen Materialien ermittelt, deren zuverlässige Bedarfsbefriedigung besonders wichtig für das Unternehmen ist. Anschließend werden deren Mindestbestände und Losgrößen in Bewertungsläufen aktualisiert. Für die Berechnung der optimalen Sicherheitsbestände werden die Aspekte Lagerverfügbarkeit, Produktionsprozesse und Beschaffung berücksichtigt. Durch die permanente und aktuelle Nachführung dieser Parameter bilden die bestandsbezogenen Stammdaten damit die jeweiligen saisonalen Besonderheiten nicht nur genauer ab, sondern können auch einen Hinweis geben, welche Prozesse verändert werden müssen, um einen hohen Servicelevel trotz hoher Ressourcenkonkurrenz zu garantieren.

\section{Fallbeispiel 5: Customer Data Platt- form für bessere Stammdaten}

Heute werden Kunden von Unternehmen auf unterschiedlichen Kanälen angesprochen, wie Social Media, Online-Shops, Webseiten und Messengerdiensten. Nicht überall erfolgt eine explizite Identifizierung des Kunden, z.B. durch eine E-Mail-Adresse oder eine Telefonnummer. Wo dieses deterministische Matching nicht möglich ist, kann ein probalisitisches Matching dennoch helfen, den Kunden z. B. anhand des verwendeten Gerätes oder des Nutzungsortes zu identifizieren. Die gesammelten Daten können in Beziehung zueinander gesetzt werden. So ist es möglich, aus Bewegungsdaten (wie z. B. dem Verlauf des Besuches in einem Online-Shop und Posts auf Social Media) Informationen für die Profilstammdaten

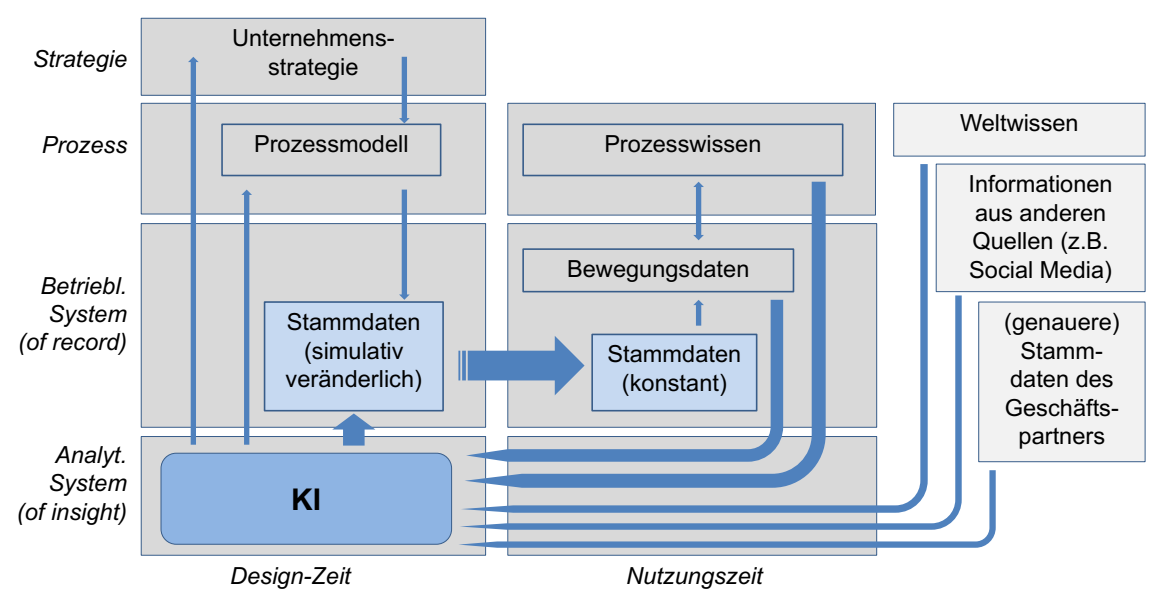

Bild 1: Vorschlag für ein Rahmenwerk zur kontinuierlichen Stammdatenverbesserung. des Kunden zu generieren. Solche Profilstammdaten sind z. B. Interessensgebiete. Anschließend sind diese zusätzlichen Stammdaten die Basis für Marketing- oder Vertriebsaktionen. Insbesondere wenn die gewonnenen Erkenntnisse neu sind, ergibt sich aus dieser Überführung von Bewegungsdaten in Stammdaten ein Potenzial für zusätzliche Umsätze.

\section{Fallbeispiel 6: Analyse von Retou- rengründen über Verkaufsartikel}

Im letzten Beispiel werden anfallende Bewegungsdaten, nämlich die Angabe des Rücksendegrundes auf dem Retourenschein, zusammengefasst und in Stammdaten, nämlich eine Eigenschaft am Artikelstamm überführt. Diese Eigenschaft "Rücksendegrund“ kann wiederum im Katalog dem Interessenten beim Einkauf angezeigt werden und dessen Kaufverhalten beeinflussen. Sieht der Interessent „63\% der Kunden empfinden, dass der Artikel zu groß ausfällt", kann er in der Bestellung möglicherweise eine Nummer kleiner wählen. Dies führt dazu, dass die Wahrscheinlichkeit einer folgenden Retoure reduziert und damit Logistikkosten vermieden werden.

\section{Verallgemeinerung}

All diese Beispiele zeigen, dass durch einen Regelkreis aus der Analyse von Bewegungsdaten die Stammdaten 
einer kontinuierlichen Verbesserung unterworfen werden können. Weil Stammdaten langfristig orientiert sind, ist dabei auf einen Ausgleich aus Stabilität und fortlaufender Optimierung zu achten. Starke Prozessschwankungen sollten vermieden werden.

Ein allgemeines Rahmenwerk sollte folgende Eigenschaften aufweisen (Bild 1):

1. Unterscheidung von Design-Zeit und Nutzungszeit: Während der Nutzungszeit sollten Stammdaten in einem definierten Zeitraum konstant gehalten werden. So werden Prozessschwankungen reduziert, Messungen von Indikatoren und deren Vergleich ermöglicht und der Forderung von ERP-Systemen bzgl. der Stammdatenpflegeprozesse genüge getan.

2. Die "Außenwelt" sollte einbezogen werden. Zusätzliches Wissen, z. B. die in Beispiel 1 erwähnten Parameter, die nur der Geschäftspartner kennt, kann zu einer Verbesserung beitragen, ebenso „Weltwissen“ über saisonale Schwankungen oder zu erwartende Logistikprobleme.

3. Prozesswissen, anfallende Bewegungsdaten, Weltwissen und Wissen der Geschäftspartner können in einem analytischen System zur Optimierung der Stammdaten analysiert und optimiert werden. KI-Systeme, insbesondere Explainable $\mathrm{Kl}$, leisten hier gute Beiträge.

4. Anhand der gewonnenen Erkenntnisse lassen sich nicht nur die Stammdaten anpassen, sondern auch Strategien und Prozessmodelle hinterfragen. Komplizierte Artikel können aus dem Sortiment entfernt oder Lieferstrategien verändert werden. Man muss nicht allein in den gegebenen Restriktionen optimieren, sondern kann Restriktionen auch auflösen.

\section{Explainable Artificial Intelligence}

Während das klassische "Nachdenken“ für den Aspekt der unbekannten Einflussparameter, wie die des Ge- schäftspartners, eine sehr erfolgreiche Methode ist, kann Explainable Al konkrete Erkenntnisse darüber liefern, welcher Parameter besonders relevant bzw. einflussreich für das Analyseergebnis war. So wird eine Rückführung der Erkenntnisse bis ins Prozessmodell und in die Strategie ermöglicht. Inzwischen gibt es viele praktische Anwendungen von tiefen neuronalen Netzen auch für unstetige technologische Zusammenhänge [5]. Die Analyse des Modells mit XAl gestattet folgende Interpretationen:

- wie sicher (wie stark) ist die getroffene Klassifikation und wie unsicher sind die verworfenen Klassifikationsalternativen, korrespondierend zu der Frage „ob man dem Ergebnis vertrauen kann",

- dominieren im Datensatz eher andere Zusammenhänge als die vom Menschen zu erkennen erhofften bzw. welche Voraussetzungen für die Vertrauenswürdigkeit sind notwendig,

- welche Bereiche oder welche Parameter der Eingabedaten waren besonders relevant für die Empfehlung der Kl, korrespondierend zur Frage „hätte man auf dieser Basis gleiche Entscheidungen getroffen".

\section{Ausblick}

Aufbauend auf den hier illustrierten Beispielen lassen sich in vielen Anwendungsdomänen weitere Ideen generieren, wie eine rollierende Analyse von Bewegungsdaten die Stammdatenqualität verbessern kann. Dann gilt es zu priorisieren, welche Daten und welche Prozesse das höchste Potenzial bieten. Es ist zu klären, welche Verantwortlichkeiten bei welchen Mitarbeitern angesiedelt werden müssen und wie man diesen Prozess im ERPSystem verankert. Wenn Stammdaten häufig angepasst werden sollen, empfiehlt sich die Automatisierung des Prozesses oder zumindest eine Softwarestützung über die Möglichkeiten des ERP-Systems hinaus, wie z.B. mit ORSOFT Master Data Workflow. Daten sind das neue Gold. Die technischen
Möglichkeiten gestatten eine Auflösung der historischen Trennung von Stamm- und Bewegungsdaten auch im ERP-Umfeld. Die Potenziale liegen auf der Hand.

Literatur:

[1] Kleppman, W.: Versuchsplanung Produkte und Prozesse optimieren, Hanser Verlag, 2020, München, ISBN: 978-3-446-46397-4

[2] Schmitt,R.: Datengetriebenes Qualitätsmanagement: Bericht zur GQW-Jahrestagung 2019 in Aachen, Springer Verlag, 2021, Berlin, ISBN 978-3-662-62441-8

[3] Kusne, A.G. et al: On-the-fly closed-loop materials discovery via Bayesian active learning nature communications Article number: 5966 (2020). https://www.nature.com/articles/s41467-020-19597-w, abgerufen am 02.04.2021

\section{Schlüsselwörter}

Fertigungsprozess, Stammdatenentwurf, Qualitätsverbesserung, Bestandsreichweite, Customer Data Plattform, Analyse von Retouren, Erklärbare KI, Kontinuierliche Stammdatenverbesserung

\section{Knowledge from transaction data}

Increasing the quality of master data is an important goal. Associated with it are lower error costs, more reliable processes, higher on-time delivery, better product quality and margin. The article illustrates some ideas how the analysis of transaction data can lead to better master data in ERP systems. A framework is set up in which various methods can be used to turn transaction data into better master data on a regular basis.

\section{Keyword:}

Production process, Master data design, Quality improvement, Inventory Reach, Customer Data Platform, Returns analysis, Explainable Al, Continuous master data improvement

\section{Kontakt:}

Prof. Dr. Dirk Schmalzried

E-Mail: dirk.schmalzried@eah-jena.de

Prof. Dr.-Ing. Siegfried Schmalzried

E-Mail: siegfried.schmalzried@

hs-furtwangen.de

Dipl.-Inform. Matthias Fiebig

E-Mail: matthias.fiebig@orsoft.de 